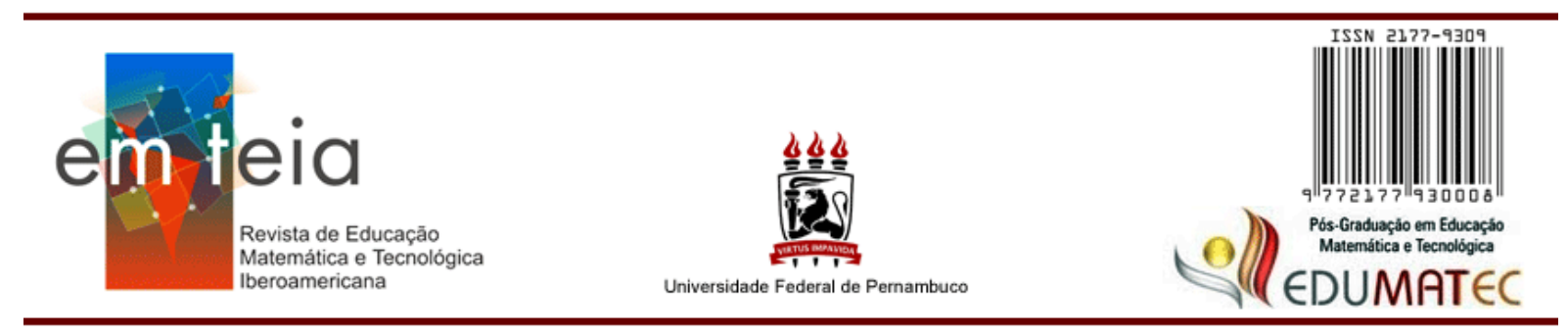

Alfabetización estadística en el currículo de matemática de Educación

\title{
Primaria en España
}

Statistical literacy in the curriculum of mathematics in primary education in Spain

Danilo Díaz-Levicoy

Departamento de Didáctica de la Matemática Universidad de Granada - Granada - España dddiaz01@hotmail.com

Karen Ruiz-Reyes

Departamento de Didáctica de la Matemática Universidad de Granada - Granada - España karenruizreyes@gmail.com

Elena Molina-Portillo

Departamento de Didáctica de la Matemática Universidad de Granada - Granada - España elemo@ugr.es

José M. Contreras García

Departamento de Didáctica de la Matemática Universidad de Granada - Granada - España jmcontreras@ugr.es

\section{Resumen}

En este artículo presentamos los resultados de un análisis sobre la alfabetización estadística en directrices curriculares de Educación Primaria nacionales (España) y comunitarias (Andalucía). La investigación ha seguido una metodología cualitativa mediante el análisis de contenido. Los resultados evidencian una presencia de variados elementos de alfabetización estadística como el uso de diferentes formas de lenguajes (natural, tabular y gráfico); la utilización de diversos contenidos matemáticos (números, geometría y proporcionalidad); la presencia de contextos personales y sociales, con actividades relacionadas al entorno inmediato del estudiante. Aspectos relacionados con actitudes, creencias y valores se observan esporádicamente, mientras que están ausentes aspectos relacionados a las emociones.

Palabras clave: Alfabetización estadística, Currículo, Educación Primaria.

\begin{abstract}
In this paper, we present the results of an analysis on statistical literacy in national (Spain) and community (Andalucía) curricular guidelines for Primary Education. The research has followed a qualitative methodology through content analysis. The results show a presence of varied elements of statistical literacy as the use of different forms of languages (natural, tabular and graphic); the use of diverse mathematical contents (numbers, geometry and proportionality); the presence of personal and social contexts, with activities related to the immediate environment of the student. Aspects related to attitudes, beliefs and values are observed sporadically, while aspects related to emotions are absent.
\end{abstract}

Keywords: Statistical literacy, curriculum, Primary Education. 


\section{Introducción}

El quehacer educativo tiene su fundamento en la búsqueda del aprendizaje de los estudiantes que participan en el proceso de instrucción, donde se espera que adquieran conocimientos, conductas y estrategias que no dominaban al iniciar un proceso de formación (RODRÍGUEZ, 2013). Para lograr este objetivo es necesario que se conjuguen adecuadamente diferentes aspectos que den sentido al proceso de enseñanza y aprendizaje, que garanticen el dominio de saberes técnicos de la disciplina y que estos sean aplicados en situaciones de la vida cotidiana. En tal sentido, la organización de los objetivos y contenidos, por medio del currículo de cada país u organización territorial, entrega una clara visión de lo que los profesores deben enseñar y lo que deben aprender los estudiantes al finalizar su formación.

Al analizar las temáticas de estadística y probabilidad vemos como su inclusión es reciente en el currículo de diferentes países (BATANERO, 2001; CAMPOS; CAZORLA; KATAOKA, 2011; FONT, 2008), situación justificada por la necesidad de conocer e interpretar situaciones y fenómenos que se representan por medio de elementos estadísticos (tablas, gráficos, medidas) en la vida cotidiana. Es así como en esta investigación nos interesa indagar sobre la presencia de elementos de alfabetización estadística en las directrices curriculares nacionales y autonómicas, en España y Andalucía respectivamente, por ser este aspecto un elemento clave en la cultura de los ciudadanos.

De acuerdo a estas consideraciones es que nos planteamos el objetivo de identificar los elementos de alfabetización estadística que subyacen en las directrices curriculares de España y Andalucía.

\section{Fundamentos}

\section{Currículo}

Currículo es una expresión que se utiliza con mucha frecuencia en el ámbito educativo. Pero, aún así, es un término que posee diferentes significados (GVIRTZ; PLAMIDESSI, 1998). Del Basto (2005) confirma esta situación al señalar que "la noción de currículo es polisémica y polimorfa, se encuentran múltiples definiciones, diferentes corrientes, posturas teóricas y variadas interpretaciones" (p. 2). El currículo presenta y organiza las estrategias para la enseñanza de diferentes temas de acuerdo a las características de la sociedad o en contexto en el que se lleva a cabo el proceso de instrucción (RODRÍGUEZ, 2013). Pinkasz y Tiramonti (2006) expresan que el currículo es el "resultado 
de un proceso de selección cultural que establece, para una sociedad en un momento determinado, qué es lo deseable que las nuevas generaciones aprendan” (p. 68).

En esta investigación compartimos y hacemos nuestra la definición de currículo entregada por Dolores (2012) al señalar que se refiere al "conjunto de objetivos, contenidos, criterios metodológicos y de evaluación que los alumnos deben alcanzar en un determinado nivel educativo. Todos estos elementos están presentes en los planes y programas de estudio [directrices curriculares]" (p. 119). Estos cuatro componentes (objetivos, contenidos, metodología, y criterios e instrumentos de evaluación) caracterizan el plan de actuación de los profesores encargados de desarrollar el proceso de instrucción (STEINER, 1980). En este mismo sentido, Coll (1992) señala que los elementos del currículo son: qué enseñar (contenidos y propósitos); cuándo enseñar (secuencia); cómo enseñar (estructura de actividades); y qué, cómo y cuándo evaluar (evaluación de la instrucción).

Posner (1998) menciona cinco tipos de currícula, entre los que se encuentran:

- Oficial. Objetivos, contenidos, criterios de evaluación descritos en los documentos oficiales a través de las directrices curriculares y que se espera alcanzar mediante el proceso de instrucción.

- Operacional. Que se implementa en el aula y que es evaluado en el proceso de formación.

- Oculto. Normas y valores que la unidad educativa desea transmitir, pero que no son reconocidos abiertamente.

- Nulo. Temas que no son abordados o aquellos temas que pertenecen al currículo y no poseen aplicabilidad ni utilidad.

- Extra currículum. Experiencias externas al currículo formal, voluntario y relacionado con los intereses de los niños y jóvenes.

De modo similar, Alsina (2000) plantea que el currículo puede ser: oficial (lo señalado en los documentos oficiales, tales como las directrices curriculares o guía para profesores), potencial (refiere a publicaciones, como los libros de texto y otros materiales didácticos), impartido (parte del currículo que el profesor efectivamente enseña en un determinado tiempo), y aprendido (aprendizajes que el estudiante adquiere durante el proceso de instrucción).

Los autores de este trabajo coinciden con otros, como Dolores (2012) y Rosales (2010), al señalar que el currículo oficial está definido mediante contenidos y capacidades que se enseñan en las unidades educativas y que se desarrollan en las clases con ayuda del 
profesor. Esta relevancia para el proceso de instrucción es la que motiva el estudio de la alfabetización estadística en el currículo oficial (o simplemente currículo) declarado en las directrices curriculares de matemática en España y Andalucía.

\section{Alfabetización estadística}

El término alfabetización estadística o cultura estadística (statistics literacy) se refiere a la importancia del conocimiento estadístico elemental en la formación de niños y jóvenes, que les permita leer e interpretar diferente información estadística a la que acceden diariamente en su trabajo, medio de comunicación o vida personal (GAL, 2002; MURRAY; GAL, 2002; WALLMAN, 1993; WATSON, 1997). Batanero (2004) señala que este término ha nacido en forma espontánea entre estadísticos y educadores estadísticos, destacando que la estadística es parte de la herencia cultural de los ciudadanos. Gal (2002) define alfabetización estadística como la unión de dos competencias relacionadas:

a) la habilidad de las personas para interpretar y evaluar críticamente la información estadística, los argumentos basados en datos, o los fenómenos estocásticos, que pueden encontrar en diversos contextos, y cuando sea relevante; y b) su habilidad para discutir o comunicar sus reacciones a la información estadística, tales como su comprensión del significado de la información, sus opiniones sobre las implicaciones de esta información, o sus preocupaciones relativas a la aceptabilidad de las conclusiones dadas (p. 2-3).

De lo anterior, algunas capacidades que debe tener una persona alfabetizada estadísticamente: comprender los conceptos y herramientas básicas de estadística; leer e interpretar datos, tablas, gráficos y medidas de resumen; visión crítica sobre el uso de la estadística en los medios de comunicación; y valorar la estadística en su vida personal, social y laboral.

\section{Directrices curriculares españolas y andaluzas}

Las directrices curriculares actuales en España se enmarcan dentro de la Ley Orgánica 8/2013, de 9 de diciembre, para la Mejora de la Calidad Educativa (LOMCE), y que se concreta, en primera instancia, con el Real Decreto 126/2014, de 28 de febrero, por el que se establece el currículo básico de la Educación Primaria (MECD, 2014).

En estas directrices curriculares los contenidos del área de matemática se organizan en cinco bloques, con el primero de ellos cuáles es transversal a los demás: 1) Procesos, métodos y actitudes en matemáticas; 2) Números; 3) Medida; 4) Geometría; 5) Estadística y probabilidad. 
En el Cuadro 1, vemos la organización del bloque 5 (Estadística y probabilidad), donde se mencionan y establecen los contenidos, criterios de evaluación y estándares de aprendizajes evaluables.

\begin{tabular}{|c|c|c|}
\hline Contenidos & Criterios de Evaluación & Estándares de aprendizajes evaluables \\
\hline 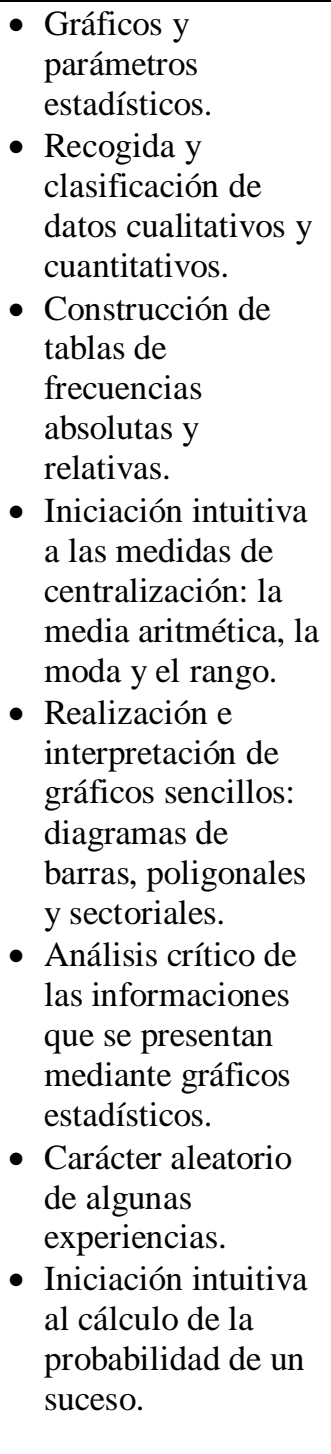 & $\begin{array}{l}\text { - Recoger y registrar una } \\
\text { información cuantificable, } \\
\text { utilizando algunos recursos } \\
\text { sencillos de representación gráfica: } \\
\text { tablas de datos, bloques de barras, } \\
\text { diagramas lineales, comunicando la } \\
\text { información. } \\
\text { - Realizar, leer e interpretar } \\
\text { representaciones gráficas de un } \\
\text { conjunto de datos relativos al } \\
\text { entorno inmediato. } \\
\text { - Hacer estimaciones basadas en la } \\
\text { experiencia sobre el resultado } \\
\text { (posible, imposible, seguro, más o } \\
\text { menos probable) de situaciones } \\
\text { sencillas en las que intervenga el } \\
\text { azar y comprobar dicho resultado. } \\
\text { Observar y constatar que hay } \\
\text { sucesos imposibles, sucesos que } \\
\text { con casi toda seguridad se } \\
\text { producen, o que se repiten, siendo } \\
\text { más o menos probable esta } \\
\text { repetición. } \\
\text { Identificar, resolver problemas de } \\
\text { la vida cotidiana, adecuados a su } \\
\text { nivel, estableciendo conexiones } \\
\text { entre la realidad y las matemáticas } \\
\text { y valorando la utilidad de los } \\
\text { conocimientos matemáticos } \\
\text { adecuados y reflexionando sobre el } \\
\text { proceso aplicado para la resolución } \\
\text { de problemas. }\end{array}$ & $\begin{array}{l}\text { - Identifica datos cualitativos y cuantitativos } \\
\text { en situaciones familiares. } \\
\text { - Recoge y clasifica datos cualitativos y } \\
\text { cuantitativos, de situaciones de su entorno, } \\
\text { utilizándolos para construir tablas de } \\
\text { frecuencias absolutas y relativas. } \\
\text { - Aplica de forma intuitiva a situaciones } \\
\text { familiares, las medidas de centralización: la } \\
\text { media aritmética, la moda y el rango. } \\
\text { - Realiza e interpreta gráficos muy sencillos: } \\
\text { diagramas de barras, poligonales y } \\
\text { sectoriales, con datos obtenidos de } \\
\text { situaciones muy cercanas. } \\
\text { - Realiza análisis crítico argumentado sobre } \\
\text { las informaciones que se presentan } \\
\text { mediante gráficos estadísticos. } \\
\text { - Identifica situaciones de carácter aleatorio. } \\
\text { Realiza conjeturas y estimaciones sobre } \\
\text { algunos juegos (monedas, dados, cartas, } \\
\text { lotería...). } \\
\text { - Resuelve problemas que impliquen } \\
\text { dominio de los contenidos propios de } \\
\text { estadística y probabilidad, utilizando } \\
\text { estrategias heurísticas, de razonamiento } \\
\text { (clasificación, reconocimiento de las } \\
\text { relaciones, uso de contraejemplos), creando } \\
\text { conjeturas, construyendo, argumentando, y } \\
\text { tomando decisiones, valorando las } \\
\text { consecuencias de las mismas y la } \\
\text { conveniencia de su utilización. } \\
\text { de problexiona sobre el proceso de resolución } \\
\text { utilizadas, las unidades de los resultados, } \\
\text { comprobando e interpretando las soluciones } \\
\text { en el contexto, proponiendo otras formas de } \\
\text { resolverlo. }\end{array}$ \\
\hline
\end{tabular}

Cuadro 1 - Descripción del Bloque 5. Estadística y probabilidad

Fuente: MECD (2014, p. 36)

La Consejería de Educación, Cultura y Deporte (CECD, 2015), por medio de la Orden de 17 de marzo de 2015, por la que se desarrolla el currículo correspondiente a la Educación

Primaria en Andalucía. La comunidad autónoma de Andalucía entrega las directrices curriculares que guiarán el proceso de instrucción de quienes cursen la primaria en este territorio. La cual complementa las directrices nacionales del MECD (2014) y que considera tres ciclos, de dos años cada uno. En los que sigue detallamos lo que los estudiantes debieran trabajar en cada ciclo que conforma la Educación Primaria.

El primer ciclo contempla la enseñanza de niños entre 6 y 7 años de edad, y los temas 
que deben trabajar, sobre estadística y probabilidad, se mencionan en el Cuadro 2.

\begin{tabular}{|c|c|c|}
\hline Criterio de evaluación & Contenidos & Indicadores \\
\hline $\begin{array}{l}\text { Leer, entender, recoger y } \\
\text { registrar una información } \\
\text { cuantificable de los contextos } \\
\text { familiar y escolar, utilizando } \\
\text { algunos recursos sencillos de } \\
\text { representación gráfica: tablas } \\
\text { de datos y diagramas de barras, } \\
\text { comunicando oralmente la } \\
\text { información }\end{array}$ & $\begin{array}{l}\text { - Gráficos estadísticos. } \\
\text { - Interpretación y construcción de tablas } \\
\text { elementales. } \\
\text { - Realización e interpretación de gráficos } \\
\text { sencillos: diagramas de barras. } \\
\text { - Utilización de técnicas elementales para la } \\
\text { recogida y ordenación de datos en } \\
\text { contextos familiares y cercanos. } \\
\text { - Descripción oral de los procedimientos de } \\
\text { registro e interpretación y resolución. } \\
\text { - Atención y cuidado en el registro de } \\
\text { información y su representación gráfica. } \\
\text { - Autoconfianza; esfuerzo y constancia en } \\
\text { la búsqueda de soluciones a situaciones } \\
\text { problemáticas construidas a partir de la } \\
\text { interpretación de gráficos y tablas. }\end{array}$ & $\begin{array}{l}\text { - Lee y entiende una } \\
\text { información cuantificable } \\
\text { de los contextos familiar } \\
\text { y escolar en tablas de } \\
\text { datos y diagramas de } \\
\text { barras comunicando } \\
\text { oralmente la información. } \\
\text { - Recoge y registra una } \\
\text { información cuantificable } \\
\text { de los contextos familiar } \\
\text { y escolar en tablas de } \\
\text { datos y diagramas de } \\
\text { barras, comunicando } \\
\text { oralmente la información. }\end{array}$ \\
\hline
\end{tabular}

Cuadro 2 - Descripción del trabajo con estadística y probabilidad en el primer ciclo de Educación Primaria

Fuente: CECD (2015, p. 260)

El segundo ciclo de Educación Primaria atiende a los estudiantes que tienen entre 8 y

9 años de edad, y los tópicos de estadística que deben trabajar se mencionan en el Cuadro 3.

\begin{tabular}{|c|c|c|}
\hline Criterio de evaluación & Contenidos & Indicadores \\
\hline $\begin{array}{l}\text { Leer e interpretar, recoger } \\
\text { y registrar una información } \\
\text { cuantificable del entorno } \\
\text { cercano utilizando algunos } \\
\text { recursos sencillos de } \\
\text { representación gráfica: } \\
\text { tablas de datos, diagramas } \\
\text { de barras, diagramas } \\
\text { lineales. Comunicar la } \\
\text { información oralmente y } \\
\text { por escrito. }\end{array}$ & $\begin{array}{l}\text { - Gráficos y parámetros estadísticos: } \\
\text { tablas de datos, diagramas de barras, } \\
\text { diagramas lineales. } \\
\text { - Recogida y clasificación de datos } \\
\text { cuantitativos utilizando técnicas } \\
\text { elementales de encuesta, observación y } \\
\text { medición. } \\
\text { - Utilización e interpretación de tablas de } \\
\text { datos, diagramas de barras, diagramas } \\
\text { lineales. } \\
\text { - Análisis de las informaciones que se } \\
\text { presentan mediante gráficos sencillos. } \\
\text { - Descripción verbal de elementos } \\
\text { significativos de gráficos sencillos } \\
\text { relativos a fenómenos familiares. } \\
\text { - Interés por el orden y la claridad en la } \\
\text { elaboración y presentación de gráficos y } \\
\text { tablas. } \\
\text { - Confianza en las propias posibilidades, } \\
\text { curiosidad, interés y constancia en la } \\
\text { interpretación de datos presentados de } \\
\text { forma gráfica. }\end{array}$ & $\begin{array}{l}\text { - Lee e interpreta una } \\
\text { información cuantificable del } \\
\text { entorno cercano utilizando } \\
\text { algunos recursos sencillos de } \\
\text { representación gráfica: tablas } \\
\text { de datos, diagramas de barras, } \\
\text { diagramas lineales, } \\
\text { comunicando la información } \\
\text { oralmente y por escrito. } \\
\text { - Registra una información } \\
\text { cuantificable del entorno } \\
\text { cercano utilizando algunos } \\
\text { recursos sencillos de } \\
\text { representación gráfica: tablas } \\
\text { de datos, diagramas de barras, } \\
\text { diagramas lineales, } \\
\text { comunicando la información } \\
\text { oralmente y por escrito. }\end{array}$ \\
\hline $\begin{array}{l}\text { - Observar que, en el entorno } \\
\text { cercano, hay sucesos } \\
\text { imposibles y sucesos que } \\
\text { con casi toda seguridad se } \\
\text { producen, hacer } \\
\text { estimaciones basadas en la } \\
\text { experiencia sobre el } \\
\text { resultado (posible, }\end{array}$ & $\begin{array}{l}\text { - Sucesos posibles y sucesos imposibles. } \\
\text { - Realización de estimaciones sobre } \\
\text { algunos juegos y sucesos. } \\
\text { - Confianza en las propias posibilidades y } \\
\text { curiosidad, interés y constancia en la } \\
\text { interpretación de datos presentados de } \\
\text { forma gráfica. } \\
\text { - Curiosidad por comparar los resultados }\end{array}$ & $\begin{array}{l}\text { - Observa que en el entorno } \\
\text { cercano hay sucesos } \\
\text { imposibles y sucesos que con } \\
\text { casi toda seguridad se } \\
\text { producen. } \\
\text { - Hacer estimaciones basadas en } \\
\text { la experiencia sobre el } \\
\text { resultado (posible, imposible) }\end{array}$ \\
\hline
\end{tabular}




\begin{tabular}{|l|l|l|}
\hline \multicolumn{1}{|c|}{ Criterio de evaluación } & \multicolumn{1}{c|}{ Contenidos } & \multicolumn{1}{c|}{ Indicadores } \\
\hline $\begin{array}{l}\text { imposible) de situaciones } \\
\text { sencillas y comprobar } \\
\text { dicho resultado. }\end{array}$ & $\begin{array}{l}\text { de las estimaciones y la realidad en } \\
\text { algunos sucesos. }\end{array}$ & $\begin{array}{l}\text { de situaciones sencillas y } \\
\text { comprobar dicho resultado. }\end{array}$ \\
\hline
\end{tabular}

Cuadro 3 - Descripción del trabajo con estadística y probabilidad en el segundo ciclo de Educación Primaria Fuente: CECD (2015, p. 276-277)

El tercer ciclo, y final de la Educación Primaria, está destinados a educar a estudiantes que se encuentran en las edades de 10 y 11 años, y cuya instrucción debe considerar los temas descritos en el Cuadro 4.

\begin{tabular}{|c|c|c|}
\hline Criterio de evaluación & Contenidos & Indicadores \\
\hline $\begin{array}{l}\text { Leer e interpretar, } \\
\text { recoger y registrar una } \\
\text { información } \\
\text { cuantificable en } \\
\text { situaciones familiares } \\
\text { del contexto social, } \\
\text { utilizando y elaborando } \\
\text { algunos recursos } \\
\text { sencillos de } \\
\text { representación gráfica: } \\
\text { tablas de datos, } \\
\text { diagramas de barras, } \\
\text { diagramas lineales, } \\
\text { diagramas poligonales y } \\
\text { sectoriales, } \\
\text { comunicando la } \\
\text { información oralmente } \\
\text { y por escrito. }\end{array}$ & $\begin{array}{l}\text { - Gráficos y parámetros estadísticos: tablas de } \\
\text { datos, diagramas de barras, diagramas lineales, } \\
\text { diagramas poligonales y sectoriales. } \\
\text { - Recogida y clasificación de datos cualitativos y } \\
\text { cuantitativos utilizando técnicas elementales de } \\
\text { encuesta, observación y medición. } \\
\text { - Construcción de tablas de frecuencias absolutas } \\
\text { y relativas. } \\
\text { - Realización e interpretación de gráficos } \\
\text { sencillos: diagramas de barras, poligonales y } \\
\text { sectoriales. } \\
\text { - Iniciación intuitiva a las medidas de } \\
\text { centralización: la media aritmética, la moda y } \\
\text { el rango } \\
\text { - Análisis crítico de las informaciones que se } \\
\text { presentan mediante gráficos estadísticos. } \\
\text { - Carácter aleatorio de algunas experiencias. } \\
\text { - Iniciación intuitiva al cálculo de la } \\
\text { probabilidad de un suceso. } \\
\text { - Valoración de la importancia de analizar } \\
\text { críticamente las informaciones que se presentan } \\
\text { a través de gráficos estadísticos. } \\
\text { - Atención al orden y la claridad en la } \\
\text { elaboración y presentación de gráficos y tablas. } \\
\text { - Interés y curiosidad por la utilización de tablas } \\
\text { y gráficos. } \\
\text { - Confianza en las propias posibilidades al } \\
\text { afrontar la interpretación y el registro de datos } \\
\text { y la construcción de gráficos. }\end{array}$ & $\begin{array}{l}\text { - Lee e interpreta una } \\
\text { información cuantificable } \\
\text { en situaciones familiares } \\
\text { del contexto social, } \\
\text { utilizando algunos recursos } \\
\text { sencillos de representación } \\
\text { gráfica: tablas de datos, } \\
\text { diagramas de barras, } \\
\text { diagramas lineales, } \\
\text { diagramas poligonales y } \\
\text { sectoriales, comunicando la } \\
\text { información oralmente y } \\
\text { por escrito. } \\
\text { Registra una información } \\
\text { cuantificable en situaciones } \\
\text { familiares del contexto } \\
\text { social, utilizando o } \\
\text { elaborando algunos } \\
\text { recursos sencillos de } \\
\text { representación gráfica: } \\
\text { tablas de datos, diagramas } \\
\text { de barras, diagramas } \\
\text { lineales, diagramas } \\
\text { poligonales y sectoriales, } \\
\text { comunicando la } \\
\text { información oralmente y } \\
\text { por escrito. }\end{array}$ \\
\hline $\begin{array}{l}\text { - Observar y constatar, en } \\
\text { situaciones de la vida } \\
\text { cotidiana, que hay } \\
\text { sucesos imposibles, } \\
\text { sucesos que con casi } \\
\text { toda seguridad se } \\
\text { producen, o que se } \\
\text { repiten, siendo más o } \\
\text { menos probable esta } \\
\text { repetición, hacer } \\
\text { estimaciones basadas en } \\
\text { la experiencia sobre el } \\
\text { resultado (posible, } \\
\text { imposible, seguro, más } \\
\text { o menos probable) de } \\
\text { situaciones en las que }\end{array}$ & $\begin{array}{l}\text { - Carácter aleatorio de algunas experiencias. } \\
\text { - 5.8. Iniciación intuitiva al cálculo de la } \\
\text { probabilidad de un suceso. } \\
\text { - Valoración de la importancia de analizar } \\
\text { críticamente las informaciones que se presentan } \\
\text { a través de gráficos estadísticos. }\end{array}$ & $\begin{array}{l}\text { - Observa y constata, en } \\
\text { situaciones de la vida } \\
\text { cotidiana, que hay sucesos } \\
\text { imposibles, sucesos que } \\
\text { con casi toda seguridad se } \\
\text { producen, o que se repiten, } \\
\text { siendo más o menos } \\
\text { probable esta repetición. } \\
\text { - Hace estimaciones basadas } \\
\text { en la experiencia sobre el } \\
\text { resultado (posible, } \\
\text { imposible, seguro, más o } \\
\text { menos probable) de } \\
\text { situaciones en las que } \\
\text { intervenga el azar y } \\
\text { comprobar dicho resultado. }\end{array}$ \\
\hline
\end{tabular}




\begin{tabular}{|l|l|c|}
\hline Criterio de evaluación & Contenidos & Indicadores \\
\hline $\begin{array}{l}\text { intervenga el azar y } \\
\text { comprobar dicho }\end{array}$ & & \\
resultado. & & \\
\hline
\end{tabular}

Cuadro 4 - Descripción del trabajo con estadística y probabilidad en el tercer ciclo de Educación Primaria Fuente: CECD (2015, p. 296-297)

\section{Antecedentes: investigaciones sobre directrices curriculares}

En este punto describiremos algunos trabajos que centran su interés en analizar y describir las directrices curriculares de matemática o estadística de educación Primaria.

Rawson, Chamoso y Rodríguez (1999) analizan el currículo de matemática en Inglaterra y España (contenidos de la educación obligatoria). Estos autores observan diferencias sobre edad y secuencia en que se trabajan algunos los contenidos (conceptuales, procedimentales y actitudinales) según el contexto de cada país.

Batanero, Contreras y Arteaga (2011) reportan el análisis de las directrices curriculares en la Educación Primaria española. Se evidencian sugerencias metodologías para trabajar actividades exploratorias y que permiten desarrollar aspectos intuitivos, y plantean desafíos para el trabajo en aula. Este trabajo es ampliado en Batanero, Gea, Arteaga y Contreras (2014), considerando en su análisis la Educación Primaria y Secundaria, así como la presencia de la estadística en evaluaciones externas. Tras este análisis se menciona que estas orientaciones siguen las corrientes internacionales, buscando formar ciudadanos estadísticamente cultos. Estas directrices curriculares, según los autores, plantean una reflexión profunda sobre el proceso de instrucción pues, en algunos niveles, se presentan contenidos complejos para las edades involucradas.

Castellanos y Arteaga (2013) estudian los gráficos estadísticos en las directrices curriculares de Educación Primaria de Colombia y España. Los resultados muestran la coincidencia sobre los temas a bordar (datos estadísticos, variables discretas, gráficos y tablas estadísticas y parámetros estadísticos) y coinciden en señalar que las tablas y gráficas estadísticas son necesarias para el estudio del tratamiento de la información.

Pino, Díaz-Levicoy y Piñeiro (2014) estudian la presencia de los gráficos estadísticos en las orientaciones curriculares de Matemática, Ciencias Naturales y Ciencias Sociales para la Educación Primaria en Chile. Como resultado se evidencia una gran variedad de actividades sobre este tema, valorando la relación que se establece entre diferentes áreas por medio de los gráficos. Confirmando que éstos permiten comprender el contexto, los cambios sociales y fenómenos naturales; favoreciendo el trabajo interdisciplinario.

Opolot-Okurut y Eluk (2011) describen el currículo para la enseñanza de la estadística 
en Educación Primaria y Secundaria de Uganda. El análisis refleja la necesidad de que el proceso de instrucción considere: el uso de herramientas tecnológicas, favorezca un aprendizaje activo y cooperativo, emplee diversidad de métodos, y aumente las investigaciones para que los educadores estadísticos se informen.

Campos, Cazorla y Kataoka (2011) estudian las directrices curriculares de la Educación Primaria de Brasil. El estudio señala que el currículo tiene un carácter innovador, para formar a ciudadanos con un espíritu crítico y una adecuada formación cívica, mediante el análisis e interpretación de los datos que permite describir el entorno y realidad del estudiante. Según los autores, algunos desafíos que estas plantean son: mejorar los libros de texto, subsanar errores conceptuales y la fragmentación de los contenidos, superar la falta de material didáctico para desarrollar el proceso de instrucción, y que las investigaciones sobre Educación Estadística lleguen a las escuelas.

Vásquez y Alsina (2014) estudian la presencia de la probabilidad en los documentos curriculares americanos (NCTM, 2000; CCSSI, 2010), currículo de Educación Primaria chilena y española, para tener claridad sobre los conocimientos que debe poseer el profesor de primaria para efectuar el proceso de instrucción. Se muestran modelos sobre el conocimiento didáctico y matemático del profesor, para garantizar mejoras en estos aspectos.

Morales y Ruiz (2013) comparan los contenidos de estadística y probabilidad en España y Chile para la Educación Primaria. La comparación permite observar coincidencias en sugerir el uso de herramientas tecnológicas para mayor comprensión de los contenidos y utilizar datos reales para trabajar las tablas y gráficas estadísticas. Dentro de las diferencias se observan que en los primeros años de la primaria chilena se trabaja con datos y representaciones gráficas de experimentos aleatorios, y en España se comienza trabajando el lenguaje ligado a la probabilidad (imposible, seguro y aquello que es posible pero no seguro). Se identifica que, en los últimos años de la Educación Primaria, en ambos países se introducen las medidas de tendencia central, y ampliando la diversidad de gráficos.

A modo de resumen, vemos como diversas directrices curriculares demandan el trabajo con elementos de estadística desde los primeros cursos de Educación Primaria, entre ellos tablas, gráficos estadísticos, medidas de resumen, probabilidad, entre otros (BATANERO; CONTRERAS; ARTEAGA, 2011; CAMPOS; CAZORLA; KATAOKA, 2011; OPOLOT-OKURUT; ELUK, 2011; VÁSQUEZ; ALSINA, 2014), y que plantea desafíos en la formación de profesores (BATANERO; CONTRERAS; ARTEAGA, 2011; CASTELlANOS; ARTEAGA, 2013; MORALES; RUIZ, 2013; VÁSQUEZ; ALSINA, 2014). 
Con este trabajo queremos ampliar la perspectiva de estudio al considerar como tema de interés la alfabetización estadística en el currículo de Educación Primaria en España y Andalucía. Tema que no ha sido investigado desde una perspectiva curricular.

\section{Metodología}

Esta investigación sigue una metodología cualitativa, mediante un análisis de contenido. López-Noguero (2002) señala que este método es una forma particular del análisis de documentos (ejemplo libros de texto o documentos curriculares).

Para esta investigación se han considerado las directrices curriculares de España y la comunidad autónoma de Andalucía, elegidas porque son recientes y se quiere verificar si atienden a las demandas internacionales relativas a la inclusión de aspectos de alfabetización estadística, como una forma de preparar a los futuros ciudadanos para su interacción en la sociedad del conocimiento y la información.

Para dicho análisis hemos considerado los componentes de alfabetización estadística que plantean en trabajos como Molina-Portillo, Contreras, Godino y Díaz-Levicoy (2017) y que corresponden a una adaptación del modelo de Gal (2002), y que observamos en el Cuadro 5.

\begin{tabular}{|c|c|}
\hline Dimensión cognitiva & Dimensión afectiva \\
\hline $\begin{array}{l}\text { Lengua natural (literacy skills): Destrezas sobre la lengua } \\
\text { natural, procesamiento textual, tabular y gráfico }\end{array}$ & $\begin{array}{l}\text { Actitudes: Orientaciones o predisposiciones } \\
\text { hacia ciertos patrones de conducta. (Adoptar } \\
\text { una posición de cuestionamiento hacia } \\
\text { mensajes cuantitativos que pueden inducir a } \\
\text { error, ser sesgados o incompletos; evaluación } \\
\text { crítica de gráficos) }\end{array}$ \\
\hline $\begin{array}{l}\text { Estadística: } \\
\text { 1) Conocer por qué se necesitan los datos y cómo se obtienen } \\
\text { 2) Familiaridad con los términos e ideas básicas sobre la } \\
\text { estadística descriptiva; } \\
\text { 3) Familiaridad con las visualizaciones gráficas, tabulares y su } \\
\text { interpretación (competencia gráfica). } \\
\text { 4) Comprensión de nociones básicas de probabilidad } \\
\text { 5) Conocer cómo se obtienen las conclusiones e inferencias } \\
\text { estadísticas }\end{array}$ & $\begin{array}{l}\text { Emociones: Estados rápidamente cambiantes } \\
\text { de sentimientos experimentados de manera } \\
\text { consciente o que ocurren de manera } \\
\text { preconsciente o inconsciente ante } \\
\text { determinadas situaciones. }\end{array}$ \\
\hline $\begin{array}{l}\text { Matemáticas: } \\
\text { 1) Números y operaciones } \\
\text { 2) Proporcionalidad } \\
\text { 3) Geometría } \\
\text { 3) Álgebra } \\
\text { 4) Funciones }\end{array}$ & $\begin{array}{l}\text { Valores: } \\
\text { Se refieren a ‘verdades personales' o } \\
\text { compromisos profundamente apreciados por } \\
\text { los individuos, incluyendo componentes } \\
\text { éticos y morales. } \\
\text { Ayudan a motivar elecciones a largo plazo o a } \\
\text { establecer prioridades a corto plazo. }\end{array}$ \\
\hline $\begin{array}{l}\text { Contextos: } \\
\text { 1) Personal } \\
\text { 2) Profesional } \\
\text { 3) Social } \\
\text { 4) Científico }\end{array}$ & $\begin{array}{l}\text { Creencias: } \\
\text { Ideas u opiniones individuales sobre un tema, } \\
\text { o dominio, sobre uno mismo, o un contexto } \\
\text { social; implican la atribución de algún tipo de } \\
\text { verdad o validez externa al sistema de } \\
\text { proposiciones u otras configuraciones } \\
\text { cognitivas. }\end{array}$ \\
\hline
\end{tabular}


Cuadro 5 - Componentes de la alfabetización estadística Fuente: Molina-Portillo et al. (2017, p. 4788)

\section{Resultados}

Los resultados del análisis de los elementos de alfabetización estadística se resumen en el Cuadro 6. En él se observa la presencia o ausencia de éstos, según la adaptación de MolinaPortillo et al. (2017). En lo que sigue describimos los resultados, para las directrices nacionales y autonómicas (por ciclo), para cada uno de estos indicadores.

\begin{tabular}{|c|c|c|c|c|c|c|c|c|c|c|c|c|c|}
\hline \multirow{2}{*}{ Variable } & \multirow{2}{*}{ Categoría } & \multicolumn{3}{|c|}{ Nacional } & \multicolumn{3}{|c|}{ I Ciclo } & \multicolumn{3}{|c|}{ II Ciclo } & \multicolumn{3}{|c|}{ III Ciclo } \\
\hline & & Con & $\mathbf{C E}$ & EA & Con & $\mathbf{C E}$ & I & Con & CE & I & Con & CE & $\mathbf{I}$ \\
\hline \multirow[t]{3}{*}{ Lenguaje } & $\begin{array}{l}\text { Lenguaje natural. } \\
\text { Cuando el enunciado } \\
\text { de la sección del libro } \\
\text { en que se usa } \\
\text { exclusivamente texto } \\
\text { (palabras y números). }\end{array}$ & $\mathrm{x}$ & $\mathrm{x}$ & $\mathrm{x}$ & $\mathrm{x}$ & $\mathrm{x}$ & $\mathrm{x}$ & $\mathrm{x}$ & $\mathrm{x}$ & $\mathrm{x}$ & $\mathrm{x}$ & $\mathrm{x}$ & $\mathrm{x}$ \\
\hline & $\begin{array}{l}\text { Tabla. Cuando el } \\
\text { enunciado está } \\
\text { basado en una tabla }\end{array}$ & $\mathrm{x}$ & $\mathrm{x}$ & $\mathrm{x}$ & $\mathrm{x}$ & $\mathrm{x}$ & $\mathrm{x}$ & $\mathrm{x}$ & $\mathrm{x}$ & $\mathrm{x}$ & $\mathrm{x}$ & $\mathrm{x}$ & $\mathrm{x}$ \\
\hline & $\begin{array}{l}\text { Gráfico estadístico. } \\
\text { Cuando el enunciado } \\
\text { está basado en un } \\
\text { gráfico }\end{array}$ & $\mathrm{x}$ & $\mathrm{x}$ & $\mathrm{x}$ & $\mathrm{x}$ & $\mathrm{x}$ & $\mathrm{x}$ & $\mathrm{x}$ & $\mathrm{x}$ & $\mathrm{x}$ & $\mathrm{x}$ & $\mathrm{x}$ & $\mathrm{x}$ \\
\hline \multirow[t]{5}{*}{$\begin{array}{l}\text { Componente } \\
\text { de la } \\
\text { Estadística }\end{array}$} & $\begin{array}{l}\text { Conocer por qué se } \\
\text { necesitan los datos y } \\
\text { cómo se obtienen }\end{array}$ & $\mathrm{x}$ & $\mathrm{x}$ & $\mathrm{x}$ & $\mathrm{x}$ & $\mathrm{x}$ & $\mathrm{x}$ & $\mathrm{x}$ & $\mathrm{x}$ & $\mathrm{x}$ & $\mathrm{x}$ & $\mathrm{x}$ & $\mathrm{x}$ \\
\hline & $\begin{array}{l}\text { Familiaridad con los } \\
\text { términos e ideas } \\
\text { básicas sobre la } \\
\text { estadística } \\
\text { descriptiva }\end{array}$ & $\mathrm{x}$ & $\mathrm{x}$ & $\mathrm{x}$ & $\mathrm{x}$ & $\mathrm{x}$ & $\mathrm{x}$ & $\mathrm{x}$ & $\mathrm{x}$ & $\mathrm{x}$ & $\mathrm{x}$ & $\mathrm{x}$ & $\mathrm{x}$ \\
\hline & $\begin{array}{l}\text { Familiaridad con las } \\
\text { visualizaciones } \\
\text { gráficas, tabulares y } \\
\text { su interpretación } \\
\text { (competencia gráfica) }\end{array}$ & $\mathrm{x}$ & $\mathrm{x}$ & $\mathrm{x}$ & $\mathrm{x}$ & $\mathrm{x}$ & $\mathrm{x}$ & $\mathrm{x}$ & $\mathrm{x}$ & $\mathrm{x}$ & $\mathrm{x}$ & $\mathrm{x}$ & $\mathrm{x}$ \\
\hline & $\begin{array}{l}\text { Comprensión de } \\
\text { nociones básicas de } \\
\text { probabilidad }\end{array}$ & $\mathrm{x}$ & $\mathrm{x}$ & $\mathrm{x}$ & & & & $\mathrm{x}$ & $\mathrm{x}$ & $\mathrm{x}$ & $\mathrm{x}$ & $\mathrm{x}$ & $\mathrm{x}$ \\
\hline & $\begin{array}{l}\text { Conocer cómo se } \\
\text { obtienen las } \\
\text { conclusiones e } \\
\text { inferencias } \\
\text { estadísticas }\end{array}$ & & & & & & & & & & & & \\
\hline \multirow{5}{*}{$\begin{array}{l}\text { Contenidos } \\
\text { de } \\
\text { matemática } \\
\text { involucrado }\end{array}$} & $\begin{array}{l}\text { Números y } \\
\text { operaciones }\end{array}$ & $\mathrm{x}$ & $\mathrm{x}$ & $\mathrm{x}$ & $\mathrm{x}$ & $\mathrm{x}$ & $\mathrm{x}$ & $\mathrm{x}$ & $\mathrm{x}$ & $\mathrm{x}$ & $\mathrm{x}$ & $\mathrm{x}$ & $\mathrm{x}$ \\
\hline & Proporcionalidad & $\mathrm{X}$ & $\mathrm{X}$ & $\mathrm{X}$ & $\mathrm{X}$ & $\mathrm{x}$ & $\mathrm{X}$ & $\mathrm{X}$ & $\mathrm{X}$ & $\mathrm{x}$ & $\mathrm{X}$ & $\mathrm{x}$ & $\mathrm{x}$ \\
\hline & Geometría & $\mathrm{x}$ & $\mathrm{x}$ & $\mathrm{x}$ & $\mathrm{x}$ & $\mathrm{x}$ & $\mathrm{x}$ & $\mathrm{x}$ & $\mathrm{x}$ & $\mathrm{x}$ & $\mathrm{x}$ & $\mathrm{x}$ & $\mathrm{x}$ \\
\hline & Álgebra & & & & & & & & & & & & \\
\hline & Funciones & & & & & & & & & & & & \\
\hline \multirow{4}{*}{$\begin{array}{l}\text { Contexto } \\
\text { PISA }\end{array}$} & Personal & & $\mathrm{x}$ & $\mathrm{X}$ & $\mathrm{x}$ & $\mathrm{x}$ & $\mathrm{X}$ & $\mathrm{x}$ & $\mathrm{x}$ & $\mathrm{X}$ & & $\mathrm{x}$ & $\mathrm{x}$ \\
\hline & Profesional & & & & & & & & & & & & \\
\hline & Social & & & & & & & & & & & $\mathrm{x}$ & $\mathrm{x}$ \\
\hline & Científico & & & & & & & & & & & & \\
\hline Actitudes y & Dar una opinión & X & & $\mathrm{X}$ & & & & & & & $\mathrm{x}$ & & \\
\hline
\end{tabular}




\begin{tabular}{|c|c|c|c|c|c|c|c|c|c|c|c|c|c|}
\hline \multirow{2}{*}{ Variable } & \multirow{2}{*}{ Categoría } & \multicolumn{3}{|c|}{ Nacional } & \multicolumn{3}{|c|}{ I Ciclo } & \multicolumn{3}{|c|}{ II Ciclo } & \multicolumn{3}{|c|}{ III Ciclo } \\
\hline & & Con & CE & EA & Con & $\mathrm{CE}$ & I & Con & CE & I & Con & $\mathbf{C E}$ & I \\
\hline Creencias & $\begin{array}{l}\text { sobre algún aspecto } \\
\text { del gráfico o tabla; } \\
\text { Identificar si el } \\
\text { gráfico presenta } \\
\text { algún error; Analizar } \\
\text { conclusiones; } \\
\text { Analizar si la } \\
\text { representación usada } \\
\text { está de acuerdo a la } \\
\text { naturaleza de los } \\
\text { datos }\end{array}$ & & & & & & & & & & & & \\
\hline Emociones & $\begin{array}{l}\text { Cuando el enunciado } \\
\text { hace referencia a una } \\
\text { situación que pueda } \\
\text { impactar a los } \\
\text { estudiantes y } \\
\text { movilizar su } \\
\text { sensibilidad. }\end{array}$ & & & & & & & & & & & & \\
\hline Valores & $\begin{array}{l}\text { Se refieren a } \\
\text { 'verdades personales' } \\
\text { o compromisos } \\
\text { profundamente } \\
\text { apreciados por los } \\
\text { individuos, } \\
\text { incluyendo } \\
\text { componentes éticos y } \\
\text { morales. } \\
\text { Ayudan a motivar } \\
\text { elecciones a largo } \\
\text { plazo o a establecer } \\
\text { prioridades a corto } \\
\text { plazo. }\end{array}$ & & & & $\mathrm{x}$ & & & $\mathrm{x}$ & & & $\mathrm{x}$ & & \\
\hline
\end{tabular}

Cuadro 6 - Elementos de alfabetización estadística en el currículo español y andaluz Fuente: Elaborado por los autores

\section{Lenguaje}

En las directrices curriculares, nacionales y autonómicas, observamos el trabajo con diferentes formas de lenguaje. En primer lugar, el lenguaje natural, que permite representar cantidades, así como el procesamiento de texto. Por ejemplo, el estándar de aprendizaje evaluable (Realiza análisis crítico argumentado sobre las informaciones que se presentan mediante gráficos estadísticos), en que se alude a la argumentación que deben hacer los estudiantes con la información mostrada en un gráfico; con ello se espera que estos puedan redactar ideas coherentes y claras, de modo que sean comprensibles para los demás.

En segundo lugar, vemos la presencia del lenguaje gráfico las directrices nacionales, en concreto, al menos, se deben trabajar con los gráficos de barras, líneas (poligonales) y sectores. Estos tres gráficos son confirmados en las directrices autonómicas.

En tercer lugar, está el lenguaje tabular, reflejado en el trabajo con: la construcción de 
tablas de frecuencias absolutas y relativas (contenidos del currículo nacional); registrar información cuantificable por medio de tablas (criterios de evaluación). Este trabajo es confirmado en las directrices de la comunidad andaluza, como se puede ver en el contenido del primer ciclo: "Interpretación y construcción de tablas elementales" (CECD, 2015, p. 260). Además, en estas últimas se menciona la tabla de datos.

\section{Componentes de la estadística}

Respecto a los componentes de la estadística, vemos la presencia constante de tres de ellos. En primer lugar, Conocer por qué se necesitan los datos y cómo se obtienen, desde las directrices nacionales se observan en dos de los estándares -Identifica datos cualitativos y cuantitativos en situaciones familiares; Recoge y clasifica datos cualitativos y cuantitativos, de situaciones de su entorno, utilizándolos para construir tablas de frecuencias absolutas y relativa-, en los que se trabaja la relación de datos, para abordar problemáticas del entorno del estudiante, y en las que se trabaje el obtener conclusiones, la toma de decisiones y se vea con claridad la forma de obtener los datos.

El segundo elemento, Familiaridad con los términos e ideas básicas sobre la estadística descriptiva, creemos que se observa en ambas directrices (nacionales y comunitarias), ya que en ellas se especifica una variedad de temas a abordar en la Educación Primaria, a saber: datos (cualitativos y cuantitativos), gráficos estadísticos (barras, líneas y sectores), tablas (datos y frecuencias), medidas de resumen (media aritmética, la moda y el rango), entre otros.

El tercer componente, Familiaridad con las visualizaciones gráficas, tabulares y su interpretación (competencia gráfica), se puede considerar un aspecto particular del punto anterior y que se observa, por ejemplo, en las directrices nacionales por medio del criterio de evaluación "Realizar, leer e interpretar representaciones gráficas de un conjunto de datos relativos al entorno inmediato" (MECD, 2014, p. 36). Del mismo modo, se observa su importancia en las directrices de la Junta de Andalucía, por medio del criterio de evaluación del segundo Ciclo (Leer e interpretar, recoger y registrar una información cuantificable del entorno cercano utilizando algunos recursos sencillos de representación gráfica: tablas de datos, diagramas de barras, diagramas lineales. Comunicar la información oralmente y por escrito (CECD, 2015, p. 276).

En cuarto lugar, está el elemento relacionado con las ideas de probabilidad, Comprensión de nociones básicas de probabilidad. Este tema se observa en los contenidos de las directrices nacionales "Iniciación intuitiva al cálculo de la probabilidad de un suceso" 
(MECD, 2014, p. 36) y detallándose en dos de los criterios de evaluación, donde el primero de ellos menciona: "Hacer estimaciones basadas en la experiencia sobre el resultado (posible, imposible, seguro, más o menos probable) de situaciones sencillas en las que intervenga el azar y comprobar dicho resultado" (MECD, 2014, p. 36). Por su parte, en las directrices autonómicas se observa que las ideas de probabilidad se trabajan a partir del segundo ciclo, donde uno de los criterios de evaluación dice: "Observar que, en el entorno cercano, hay sucesos imposibles y sucesos que con casi toda seguridad se producen, hacer estimaciones basadas en la experiencia sobre el resultado (posible, imposible) de situaciones sencillas y comprobar dicho resultado" (CECD, 2015, p. 277).

Finalmente, el elemento Conocer cómo se obtienen las conclusiones e inferencias estadísticas es uno de los no observados en el estudio de las directrices curriculares. Si bien, en algunos casos, se pueden observar aspectos de análisis crítico y de formulación de conclusiones no se vislumbra con claridad la forma en que estas se obtienen.

\section{Contenidos matemáticos}

Son diversos los contenidos que se encuentran detrás del trabajo con elementos estadísticos en las directrices de Educación Primaria (nacionales y autonómicas). Por ejemplo, en una tarea de construir un gráfico de sectores intervienen la idea de frecuencias y la suma de ellas (números y operaciones), la relación entre la frecuencia y la amplitud angular (proporcionalidad) y círculo y sector circular (geometría), entre otros. No observamos relación con los temas de álgebra y funciones.

\section{Contextos PISA}

Los contextos establecidos por PISA son otros de los elementos que considera la alfabetización estadística (MOLINA-PORTILLO et al., 2017). Por ejemplo, en el currículo nacional se observa el contexto personal en el criterio de evaluación "Realizar, leer e interpretar representaciones gráficas de un conjunto de datos relativos al entorno inmediato" (MECD, 2014, p. 36), ya que éste habla del entorno común del estudiante. Esto se confirma en los estándares de aprendizaje evaluables, al indicar "Aplica de forma intuitiva a situaciones familiares, las medidas de centralización: la media aritmética, la moda y el rango" (MECD, 2014, p. 36).

En las directrices de la comunidad se menciona, en cada aspecto considerado (contenidos, criterios de evaluación e indicadores), la presencia del contexto personal, excepto en el ciclo tres. Por ejemplo, en el segundo ciclo se menciona: "Descripción verbal de 
elementos significativos de gráficos sencillos relativos a fenómenos familiares" (CECD, 2015, p. 276). El contexto social, solamente se observa explícito en el ciclo tres en los criterios de evaluación y en los indicadores, el primero de ellos menciona: "Leer e interpretar, recoger y registrar una información cuantificable en situaciones familiares del contexto social, utilizando y elaborando algunos recursos sencillos de representación gráfica: tablas de datos, diagramas de barras, diagramas lineales, diagramas poligonales y sectoriales, comunicando la información oralmente y por escrito" (CECD, 2015, p. 296).

El contexto profesional y científico no se observan a simple vista.

\section{Actitudes y creencias}

Sobre las actitudes y creencias, en el currículo nacional, las vemos mencionadas en el trabajo con contenidos y estándares de aprendizajes evaluables. El primero de ellos hace referencia al "Análisis crítico de las informaciones que se presentan mediante gráficos estadísticos" (MECD, 2014, p. 36), lo que permite observar errores en la construcción de gráficos o en las conclusiones realizadas. En el segundo se menciona: "Resuelve problemas que impliquen dominio de los contenidos propios de estadística y probabilidad, utilizando estrategias heurísticas, de razonamiento (clasificación, reconocimiento de las relaciones, uso de contraejemplos), creando conjeturas, construyendo, argumentando, y tomando decisiones, valorando las consecuencias de las mismas y la conveniencia de su utilización" (MECD, 2014, p. 36), donde lo argumentativo tiene relación con entregar opiniones fundadas sobre aspectos o información del objeto matemático trabajado.

Sobre el currículo autonómico, solo se indica en los contenidos del tercer ciclo, donde se explicita el "Análisis crítico de las informaciones que se presentan mediante gráficos estadísticos" (CECD, 2015, p. 296), por medio del análisis de la forma en que se representan los datos y las conclusiones obtenidas.

\section{Emociones}

Un elemento que no observamos en las directrices (nacionales y autonómicas) son las emociones, ya que no se explicitan aspectos que movilizan la sensibilidad del estudiante. Creemos que este aspecto puede ser abordado en clases al trabajar temas actuales y de interés para los estudiantes, más allá de su color o fruta favorita, es decir, que impacte al estudiante.

\section{Valores}

Finalmente, los valores solo los vemos en los contenidos de las directrices 
autonómicas. En el primer ciclo se hace referencia a "Autoconfianza; esfuerzo y constancia en la búsqueda de soluciones a situaciones problemáticas construidas a partir de la interpretación de gráficos y tablas" (CECD, 2015, p. 260). En el segundo ciclo se trabaja la "Confianza en las propias posibilidades, curiosidad, interés y constancia en la interpretación de datos presentados de forma gráfica" (CECD, 2015, p. 276). Situación similar a lo que ocurre en el tercer ciclo.

\section{Conclusión}

Este tipo de investigaciones nos permite conocer qué se debiera enseñar en las escuelas y visualizar las posibles competencias que se quiere formar desde las aulas, qué tipo de ciudadanos se espera formar y los contenidos que deben saber los profesores antes de egresar de las instituciones formadoras.

En el caso de la alfabetización estadística, nos permite conocer cuál es la importancia que tiene este tema en las directrices curriculares de España y Andalucía, y qué elementos no están presentes y que los profesores también deben considerar en el proceso de instrucción.

Con los resultados podemos mencionar que los distintos tipos de lenguaje están explícitamente detallados, tanto en las directrices nacionales como en las autonómicas. Situación que nos parece positiva, ya que estos elementos suelen estar presentes en diferentes instancias de la vida cotidiana, y que su dominio adecuado es importante.

Sobre los componentes de la estadística, un aspecto que aparece en menor medida es obtener inferencias, aunque si se propone realizar un análisis crítico de la información trabajada, como también la elaboración de conclusiones a partir de los datos analizados. Si bien no esperamos que en Educación Primaria se trabaje con elementos de inferencia, esperamos que se aproximen a las ideas fundamentales.

Otro de los elementos considerados son los temas de matemática que se movilizan al desarrollar actividades estadísticas. Estos son diversos, ya que para comprender una medida o una representación gráfica se ocupa objetos ostensivos y no ostensivos. En esta revisión solo hemos podido identificar aquellos relacionados con los números, la proporcionalidad y la geometría, pero que se van diversificando según los temas que se trabajen y se avance en los cursos.

Los aspectos relacionados a los valores, actitudes y creencias, y emociones no se observan con tanta claridad en las directrices curriculares, incluso las últimas no se identifican. Pero, esto no significa que no intervengan en el trabajo con estadística, solo que se observa con mayor claridad en otros contextos, como los libros de texto o durante el 
proceso de instrucción; por lo que sería de interés estudiarlos.

Este trabajo es la primera aproximación al análisis de la alfabetización estadística en el currículo de Educación Primaria, motivando su ampliación en los libros de texto para tener una visión más clara sobre el significado pretendido sobre este tema.

\section{Agradecimientos}

Proyecto FCT-16-10974

\section{Referencias}

ALSINA, C. Mañana será otro día: un reto matemático llamado futuro. In: GOÑI, J. (Ed.) El currículum matemático en los inicios del siglo XXI. Barcelona: Graó, 2000. p. 13-21.

BATANERO, C. Didáctica de la Estadística. Granada: Grupo de Investigación en Educación Estadística de la Universidad de Granada, 2001.

BATANERO, C. Los retos de la cultura estadística. Yupana, Santa Fe, v. 1, n. 1, p. 27-37, 2004.

BATANERO, C.; CONTRERAS, J. M; ARTEAGA, P. El currículo de estadística en la enseñanza obligatoria. EM-TEIA. Revista de Educaçao Matematica e Tecnologica Iberoamericana, Recife, v. 2, n. 2, p. 1-20, 2011.

BATANERO, C.; GEA, M. M.; ARTEAGA, P.; CONTRERAS, J. M. La estadística en la educación obligatoria: Análisis del currículo español. Revista digital Matemática, Educación e Internet, v. 14, n. 2, p. 1-14, 2014.

CAMPOS, T.; CAZORLA, I.; KATAOKA, V. Statistics school curricula in Brazil. In: BATANERO, C.; BURRILL, G.; READING, C. (Eds.). Teaching statistics in school mathematics- Challenges for teaching and teacher education. New York: Springer, 2011. p. 5-8.

CASTELLANOS, M. T.; ARTEAGA, P. Los gráficos estadísticos en las directrices curriculares para la Educación Primaria en España y Colombia. In: CONTRERAS, J. M.; CAÑADAS, G. R.; GEA, M. M.; ARTEAGA, P. (Eds.). Actas de las Jornadas Virtuales en Didáctica de la Estadística, Probabilidad y Combinatoria. Granada: Departamento de Didáctica de la Matemática de la Universidad de Granada, 2013. p. 397-404.

CCSSI. Common Core State Standards for Mathematics. Washington, DC: National Governors Association for Best Practices and the Council of Chief State School Officers, 2010.

CECD. Consejería de Educación, Cultura y Deporte, España. Orden de 17 de marzo de 2015, por la que se desarrolla el currículo correspondiente a la Educación Primaria en Andalucía. Sevilla: Boletín Oficial de la Junta de Andalucía, 2015. 
COLL, C. Psicología y currículum. México D. F.: Paidós, 1992.

DEL BASTO, L. Reflexión sobre el currículo universitario desde la teoría discursiva de la educación. Revista ieRed. Revista Electrónica de la Red de Investigación Educativa, Popayán, v. 1, n. 3, p. 1-11, 2005.

DOLORES, C. ¿Hacia dónde reorientar el currículum de matemáticas del Bachillerato? In: DOLORES, C.; GARCÍA, M.S. (Eds.). ¿Hacia dónde reorientar el currículum de matemáticas del Bachillerato?. México: Plaza y Valdez, 2012. p. 118-133.

FONT, V. Enseñanza de la Matemática. Tendencias y perspectivas. In: GAITA, C. (Ed.). Actas III Coloquio Internacional sobre Enseñanza de las Matemáticas. Lima: Pontificia Universidad Católica del Perú, 2008. p. 21-64.

GAL, I. Adults'statistical literacy: Meanings, components, responsibilities. International Statistical Review, The Netherlands, v. 70, n. 1, p. 1-51, 2002.

GVIRTZ, S.; PLAMIDESSI, M. EI ABC de la tarea docente: currículum y enseñanza. Buenos Aires: Aique, 1998.

LÓPEZ-NOGUERO, F. El análisis de contenido como método de investigación. XXI. Revista de Educación, Huelva, n. 4, p. 167-180, 2002.

MECD. Ministerio de Educación, Cultura y Deporte, España. Real Decreto 126/2014, de 28 de febrero, por el que se establece el currículo básico de la Educación Primaria. Madrid: Boletín Oficial del Estado, 2014.

MOLINA-PORTILLO, E.; CONTRERAS, J. M.; GODINO, J. D.; DÍAZ-LEVICOY, D. Interpretación crítica de gráficos estadísticos incorrectos en la sociedad de la comunicación: un desafío para futuros maestros. Enseñanza de las Ciencias, Barcelona, n. extraordinario, p. 4787-4792, 2017.

MORALES, R.; RUIZ-REYES, K. Comparación entre los contenidos del currículo chileno y español en el área de estadística y probabilidad. In: CONTRERAS, J. M.; CAÑADAS, J. M.; GEA, M. M.; ARTEAGA, P. (Eds.). Actas de las Jornadas Virtuales en Didáctica de la Estadística, Probabilidad y Combinatoria. Granada: Departamento de Didáctica de la Matemática de la Universidad de Granada, 2013. p. 137-142.

MURRAY, S.; GAL, I. Preparing for diversity in statistics literacy: Institutional and educational implications. In: PHILLIPS, B. (Ed.). Proceedings of the Sixth International Conference on Teaching of Statistics . Ciudad del Cabo: IASE, 2002. p. 1-8.

NCTM. Principles and standards for school mathematics. Reston, VA: Autor, 2000.

OPOLOT-OKURUT, C.; ELUK, P. Statistics school curricula in Uganda. In: BATANERO, C.; BURRILL, G.; READING, C. (Eds.). Teaching statistics in school mathematics. 
Challenge for teaching and teacher education. A Joint ICMI/IASE Study. New York, NY: Springer, 2011. p. 15-19.

PINKASZ, D.; TIRAMONTI, G. Las oportunidades educativas de las mujeres en la modernización de los 90 en Argentina. In: PROVOSTE, P. (Ed.). Equidad de género y reformas educativas. Argentina, Chile, Colombia y Perú. Santiago: Hexagrama Consultoras, FLACSO y IESCO, 2006. p. 51-97.

PINO, C.; DÍAZ-LEVICOY, D.; PIÑEIRO, J. L. Los gráficos estadísticos como articuladores del currículo escolar. Revista Chilena de Educación Científica, Santiago, v. 13, n. 2, p. 9$18,2014$.

POSNER, G. Análisis de currículum. Bogotá: McGraw-Hill, 1998

RAWSON, W. B.; CHAMOSO, J. M.; RODRÍGUEZ, M. J. Los contenidos de matemáticas en la enseñanza obligatoria de Inglaterra y España. Análisis comparado. SUMA. Revista sobre la Enseñanza y el Aprendizaje de las Matemáticas, Badalona, v. 32, p. 35-46, 1999.

RODRÍGUEZ, C. El potencial curricular de los libros de texto para generar experiencias de aprendizaje. Revista Educación, San Pedro, v. 37, n. 1, 119-129, 2013.

ROSALES, J. L. Los discursos sobre el cuerpo en el currículo oficial: una aproximación al Diseño Curricular Nacional. Revista Educación, Lima, v. 19, n. 37, p. 47-66, 2010.

STEINER, H. Comparative Studies of Mathematics Curricula. Change and Stability 19601980. Bielefeld: Universität Bielefeld, 1980.

VÁSQUEZ, C.; ALSINA, Á. Enseñanza de la Probabilidad en Educación Primaria. Un Desafío para la Formación Inicial y Continua del Profesorado. Números. Revista de Didáctica de las Matemáticas, La Laguna, n. 85, p. 5-23, 2014.

WALLMAN, K. K. Enhancing statistical literacy: Enriching our society. Journal of the American Statistical Association,Virginia, n. 88, p. 1-8, 1993.

WATSON, J. Assessing statistical literacy through the use of media surveys. In: GAL, I.; GARFIELD, J. B. (Eds.). The assessment challenge in statistics education. Amsterdam: IOS Press, 1997. p. 107-121. 